\title{
Formation and Stability of Self-Assembled Coherent Islands in Highly Mismatched Heteroepitaxy
}

\author{
L. G. Wang, P. Kratzer, M. Scheffler, and N. Moll* \\ Fritz-Haber-Institut der Max-Planck-Gesellschaft, Faradayweg 4-6, D-14195 Berlin-Dahlem, Germany
}

(Received 21 December 1998)

\begin{abstract}
We study the energetics of island formation in Stranski-Krastanow growth within a parameter-free approach. It is shown that an optimum island size exists for a given coverage and island density if changes in the wetting layer morphology after the 3D transition are properly taken into account. Our approach reproduces well the experimental island size dependence on coverage and indicates that the critical layer thickness depends on growth conditions. The present study provides a new explanation for the (frequently found) rather narrow size distribution of self-assembled coherent islands. [S0031-9007(99)09155-3]
\end{abstract}

PACS numbers: $68.65 .+\mathrm{g}, 68.55 .-\mathrm{a}, 81.10 . \mathrm{Aj}$

The surface morphology of overlayers in heteroepitaxial growth has attracted intense interest because of its importance for basic science and applications in optoelectronic devices. Experiments [1-5] showed that heteroepitaxy in systems with a lattice constant difference $\geq 2 \%$, such as InAs/GaAs [2-4], Ge/Si [1], and InP/InGaP [5], follows the so-called Stranski-Krastanow growth mode [6]: three-dimensional (3D) dislocation-free (so-called coherent) islands form on top of the wetting layer. These small coherent islands are often found to have a very narrow size distribution [2-4] and are promising to be used in quantum dot light emitting diodes and lasers.

It is commonly agreed that the energetics of strain relief plays a key role in the growth process: Islands form, instead of a uniformly strained, epitaxial film, because the gain of elastic relaxation energy in an island overcompensates the cost due to the increased surface energy by islanding. It is tempting to attribute the observed island size distribution to a minimum of the free energy of the system. However, an equilibrium theory with only two energetic contributions, a positive one from the island surface energy $\left(E \sim V^{2 / 3} ; V\right.$ is the quantum dot volume) and a negative one from the elastic relaxation energy $(E \sim V)$, fails to predict a finite equilibrium size of the islands. Instead, the energy gain from strain relief always prevails for sufficiently high coverages, rendering larger islands more stable than smaller ones. In order to cope with this difficulty, several additional effects, e.g., contributions from intrinsic surface stress or from interactions between islands [7,8], have been invoked. Priester and Lannoo [9] proposed a mechanism in which 2D platelets act as precursors for the formation of 3D coherent islands, thus determining their size. Most recently, the observation of island ripening [10] has made it doubtful if the islands can be interpreted at all as structures in total equilibrium.

In this Letter, we show that the narrow size distribution of the coherent islands can be understood as the result of the system being trapped in a constrained equilibrium state, where the size is determined by the island density and the nominal coverage. In the constrained equilibrium theory, the existing nuclei grow to a size determined by the energetic balance that governs material transport between the wetting layer and the islands. This allows us to derive an optimum islands size for a fixed coverage and island density from a parameter-free approach. The elastic energy in both the islands and the substrate is calculated within continuum elasticity theory. Using densityfunctional theory within the local-density approximation, accurate surface energies are obtained with the help of the plane-wave pseudopotential method [11] for both the island facets and the wetting layer. Previous studies [12,13] had missed the latter contribution, assuming that the surface energy of the wetting layer would be unchanged by the 3D transition. Our theory reproduces very well experimental data for the island size dependence on coverage. In particular, we improve over previous approaches by showing that the selectivity of growth of a certain island size can be explained without invoking delicate elastic edge effects or island interactions $[7,8]$. Furthermore, we demonstrate how the critical layer thickness depends on growth conditions, settling this long-standing issue.

We propose a view of the growth process divided in three phases: an early nucleation phase which mainly determines the island density $n$, a second phase where the islands grow mostly on the expense of the wetting layer, and a third phase characterized by Ostwald ripening. Since we are mostly interested in island sizes, we concentrate on the second phase, and briefly discuss the third phase later. As long as the wetting layer acts as a source for material, existing nuclei will grow rapidly. Hereby the island density $n$ remains constant [3]. We treat it as an input to our model noting that the island density is determined by the growth kinetics. Furthermore, we assume the islands have identical shape and volume $V$. In the following, we discuss the island size in terms of a constrained thermodynamic equilibrium between the islands and the wetting layer, for a fixed island density. 
Although our approach is not limited to a certain system, here we consider, as an example, the strained $\{110\}$ pyramidal shaped, dislocation-free InAs islands with a square base (area $a^{2}$ ) on the GaAs(100) surface (with a wetting layer). We will also discuss the $\{111\} /\{\overline{1} \overline{1} \overline{1}\}$ faceted pyramidal islands later. We choose this system with a lattice mismatch $\Delta a \approx 7 \%$, because a large number of experimental and theoretical studies have been done [2-4,9]. The real island shape may be more complex, but the simple island shape used here should still allow us to capture the important features of the island formation (see below and Ref. [13]). Figure 1 schematically illustrates the island formation on the substrate surface. $\theta_{0}$ and $\theta$ are the nominal coverage and wetting layer thickness after island formation, respectively. We omit the interaction between islands (as we will discuss below this is a very good approximation in our case), as well as the energies of edges and corners. The entropic contribution to free energy is also neglected.

The total energy gain per unit volume of a single island can be expressed as

$$
\begin{aligned}
E_{\mathrm{tot}} / V= & \epsilon_{\mathrm{is}}^{\mathrm{el}}-\epsilon_{\mathrm{film}}^{\mathrm{el}}+\left[S \gamma_{f}-a^{2} \gamma_{\mathrm{wl}}\left(\theta_{0}\right)\right] / V \\
& +\left(1 / n-a^{2}\right) \times\left[\gamma_{\mathrm{wl}}(\theta)-\gamma_{\mathrm{wl}}\left(\theta_{0}\right)\right] / V,
\end{aligned}
$$

where $\epsilon_{\mathrm{is}}^{\mathrm{el}}$ and $\epsilon_{\mathrm{film}}^{\mathrm{el}}$ are the elastic energy densities of the island and uniformly strained film. The third term describes the change in surface energy due to the island, with $\gamma_{f}$ being the surface energy of the island facets and $S$ their area. The fourth term accounts for the thinning of the part of the wetting layer which feeds the island. $\gamma_{\mathrm{wl}}\left(\theta_{0}\right), \gamma_{\mathrm{wl}}(\theta)$ are the formation energy of the wetting layer as a function of its thickness $\theta$, measured relative to InAs bulk kept at the GaAs lattice constant. This allows us to introduce the elastic contribution to the formation energy as a uniquely defined separate term $\epsilon_{\mathrm{film}}^{\mathrm{el}}$, similar to earlier work [14]. From mass conservation, the volume of an island $V$ is given by $V=\frac{1}{6} a^{3} \tan \alpha=\frac{1}{n}\left(\theta_{0}-\theta\right) L$, where $\alpha$ and $L$ are the tilt angle of island facets and the monolayer (ML) thickness, respectively. Equation (1) holds true as long as the island contains a sufficiently high number of atoms (e.g., 5000 atoms) because elasticity theory is applicable and the reconstructions on the facets are completed [14].

In order to obtain accurate values for the surface energies and intrinsic surface stresses, these are computed

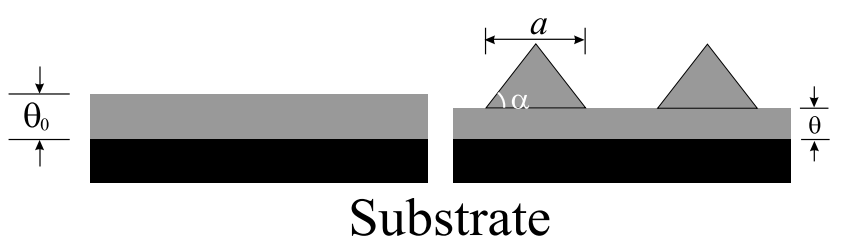

FIG. 1. Schematic illustration of the formation of coherent islands on the substrate surface. $\theta_{0}$ and $\theta$ are the nominal coverage and wetting layer thickness, respectively. $\alpha$ is the tilt angle of island facets and $a$ is the island base length.
TABLE I. Surface energies $\gamma_{f}$ and surface stresses $\sigma_{x}, \sigma_{y}$ for InAs surface reconstructions with the chemical potential $\mu_{\mathrm{As}}=\mu_{\mathrm{As}(\text { bulk })}-0.2 \mathrm{eV}$.

\begin{tabular}{clccc}
\hline \hline Surface & & $\begin{array}{c}\gamma_{f} \\
\left(\mathrm{meV} / \AA^{2}\right)\end{array}$ & $\begin{array}{c}\sigma_{x} \\
\left(\mathrm{meV} / \AA^{2}\right)\end{array}$ & $\begin{array}{c}\sigma_{y} \\
\left(\mathrm{meV} / \AA^{2}\right)\end{array}$ \\
\hline$(110)$ & Cleavage & 41 & 26 & 54 \\
$(111)$ & In vacancy & 42 & 48 & 48 \\
$(\overline{1} \overline{1} \overline{1})$ & As trimer & 49 & 92 & 92 \\
\hline \hline
\end{tabular}

using slab models of the surfaces with the help of the pseudopotential plane-wave method [11]. After optimizing the atomic geometries using consistently calculated forces on the atoms, the total energies of the slabs are computed, and the formation energies of various surfaces are obtained by subtracting the calculated total energy of an appropriate amount of bulk material. We further take into account the surface stress contribution to the surface energy up to the linear term [14], and a term proportional to the chemical potential $\mu_{\mathrm{As}}$ of the environment in case of nonstoichiometric surfaces. Since epitaxial growth is mostly performed under As-rich conditions, all surface energies are evaluated close to equilibrium with bulk arsenic (i.e., $\mu_{\mathrm{As}}=\mu_{\mathrm{As}(\mathrm{bulk})}-0.2 \mathrm{eV}$ ). For each facet, we have selected the reconstruction with the lowest energy from several candidates [14]. For the wetting layer, we consider the $\beta 2(2 \times 4)$ reconstruction which is usually found on $\mathrm{GaAs}(001)$ and $\operatorname{InAs}(001)$ surfaces under moderately As-rich conditions [15]. The results are given in Table I and Fig. 2.

The elastic energy is calculated within continuum elasticity theory using the experimental elastic moduli to describe the elastic properties of both the island and

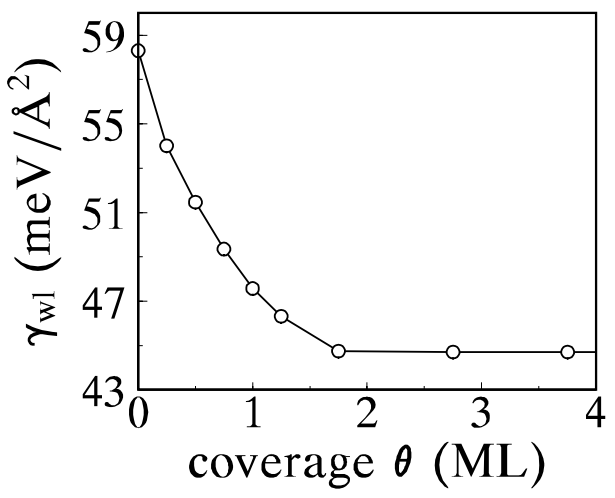

FIG. 2. Formation energy of the wetting layer as a function of thickness $\theta$, defined by $\gamma_{\mathrm{wl}} A=E^{\text {tot }}-\mu_{\mathrm{GaAs}(\mathrm{bulk})} N_{\mathrm{Ga}}-$ $\mu_{\text {InAs(bulk) }}^{\text {strained }} N_{\text {In }}-\mu_{\mathrm{As}}\left(N_{\mathrm{As}}-N_{\mathrm{Ga}}-N_{\text {In }}\right)$, where $A$ is the surface area and $N_{i}(i=\mathrm{As}, \mathrm{Ga}$, and In) are the number of particles of the species $i$ in the supercell. $E^{\text {tot }}$ is the total energy of the supercell. $\mu_{\mathrm{GaAs}(\mathrm{bulk})}$ and $\mu_{\mathrm{InAs} \text { (bulk) }}^{\text {strained }}$ are the chemical potential of GaAs bulk and of strained InAs bulk with the theoretical equilibrium lattice constant of GaAs bulk. From various configurations with $N_{\text {In }}=8 \theta$ In atoms per $(2 \times 4)$ surface unit cell, those with the lowest formation energy are presented. 
the substrate. For the island plus a $240 \AA$ thick slab representing the substrate, a finite-element approach is applied to solve the nonlinear elasticity problem [14,16]. We calculate the elastic energy for several island shapes (different tilt angles $\alpha$ of island facets) with a fixed island volume. For each particular shape, we can extract $\epsilon_{\text {is }}^{\mathrm{el}}$ from the observed linear scaling relation with the island volume. The elastic energy density $\epsilon_{\mathrm{fi} i m}^{\mathrm{el}}$ of the uniformly strained film can be obtained by extrapolating the results for the islands to $\alpha=0$, and we find it is in very good agreement with the value from linear elasticity theory.

In Fig. 3, we show the various energy contributions and the total energy gain per volume for $n=10^{10} \mathrm{~cm}^{-2}$, $\theta_{0}=1.8 \mathrm{ML}$. The elastic relaxation energy [the first and second term in Eq. (1)] is negative due to strain relief and scales linearly with the island volume. Surface energy [the third term in Eq. (1)] is a cost, and therefore its contribution is positive. The wetting layer energy contribution [the fourth term in Eq. (1)] is also positive and depends complexly on the island volume, island density, and coverage. We also show the energy contribution of the edges in Fig. 3, which becomes negligible compared to the other contributions for a large island (estimated as in Ref. [14]). It is important that an energy minimum exists in the total energy gain curve. This indicates an optimum island size can be obtained under certain growth conditions. The minimum in Fig. 3 corresponds to an island with about 38000 atoms, which compares reasonably well with typical experimental values (between 20000 [3] and 50000 atoms per island [17]). The quite uniform islands prior to ripening observed in Ref. [10] lend further support to the existence of an optimum island size. However, the island size strongly depends on the island density. Figure 4(a) shows that the equilibrium island volume $V$ is a hyperbolic function of island density $n$. As the experimental observations [18] have shown, the island radius varies exponentially with the growth temperature. This was attributed to a kinetically

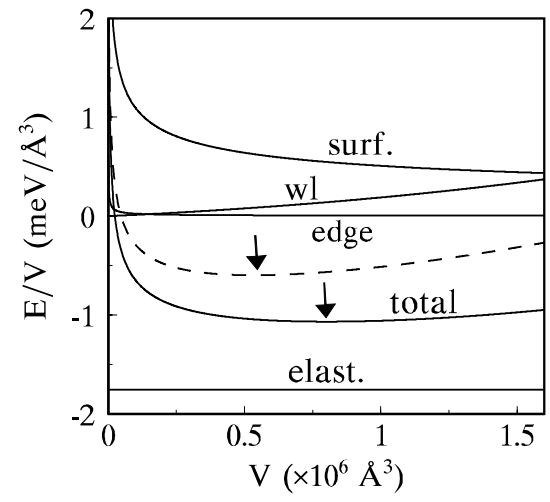

FIG. 3. Total energy gain by islanding and various energy contributions (solid lines) for $n=10^{10} \mathrm{~cm}^{-2}, \theta_{0}=1.8 \mathrm{ML}$. The dashed line is the total energy gain for $n=10^{10} \mathrm{~cm}^{-2}$, $\theta_{0}=1.5 \mathrm{ML}$. The arrows mark the minima of the curves. limited process [18]. It is consistent with the theory presented here, since the island density is known to depend strongly on the growth temperature [19,20], and thus kinetics controls the growth through controlling the island nucleation density. Figure 4(b) shows that our theory can reproduce very well the increase of the island radius with the amount of deposited material observed in various experiments, by using suitable island densities as input. The good agreement between theory and experiment also justifies the neglect of repulsive interactions between islands in the present study. A careful check indicates that the distances between the islands are quite large (larger than 65 and $220 \mathrm{~nm}$ for the high $\left[2.3 \times 10^{10} \mathrm{~cm}^{-2}\right]$ and low $\left[1.9 \times 10^{9} \mathrm{~cm}^{-2}\right]$ island densities, respectively). The island density $2.3 \times 10^{10} \mathrm{~cm}^{-2}$ used to fit the experimental results [21] agrees well with the experimentally estimated island density $1.5-2 \times 10^{10} \mathrm{~cm}^{-2}$.

The critical layer thickness is a very interesting issue and the reported values vary from 1.2 to $2 \mathrm{ML}$ [2123]. Our present theory puts us in position to discuss the critical layer thickness, because the total energy gain depends on the coverage (see Fig. 3). When we deposit less material, keeping $n$ fixed, the energy minimum rises above zero, i.e., island formation is no longer favorable. We take the critical layer thickness as the coverage at which the minimum energy equals zero (the error $\pm 0.01 \mathrm{ML}$ ). Our results, in Fig. 5, indicate that the critical layer thickness varies from 1.20 to 1.79 ML when the island density varies from $10^{9} \mathrm{~cm}^{-2}$ to $3.5 \times 10^{11} \mathrm{~cm}^{-2}$. Our theoretical prediction matches the experimentally observed range (1.2-2 ML). We note
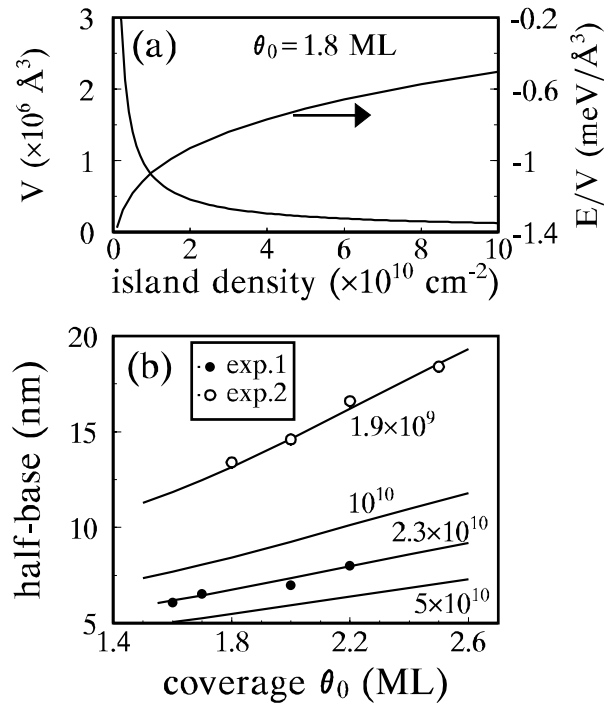

FIG. 4. The dependence of the optimum island size (volume and half-base) on the island density and the nominal coverage. In (a), the total energy gains for the optimum island size in various island densities are also shown. The experimental values in (b) are taken from Ref. [21] (•) and estimated from Ref. [3] (0). 


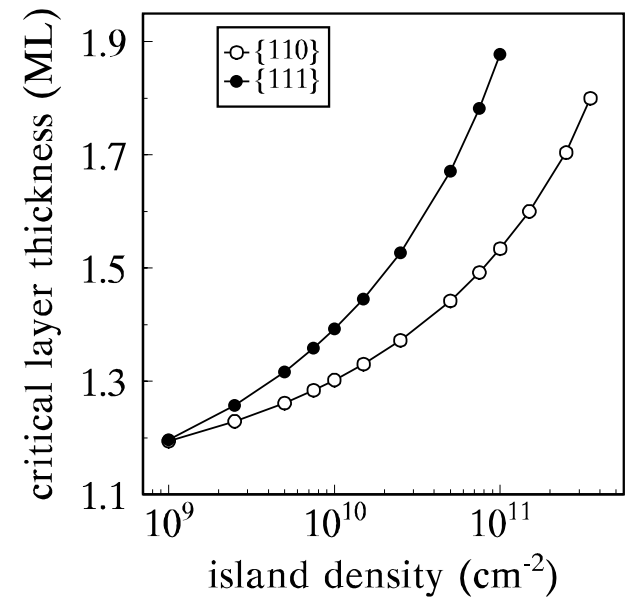

FIG. 5. Critical layer thickness as a function of the island densities.

that an exact determination is difficult, because in the experiment various other factors may influence the critical layer thickness, such as more complex island shapes, details of the growth method, and growth conditions (e.g., III/V ratio) $[24,25]$, or a possible correlation between island density and coverage [2].

We have also performed a corresponding analysis for the strained $\operatorname{InAs}\{111\} /\{\overline{1} \overline{1} \overline{1}\}$ pyramidal islands. The results show similar behavior as for the InAs $\{110\}$ islands and indicate that the basic features of our model do not depend on shape assumptions. The calculations also indicate that a larger island tends to be a steeper one, i.e., a $\{111\} /\{\overline{1} \overline{1} \overline{1}\}$ faceted pyramidal island, due to enhanced elastic energy relief $[14,26]$. Our study with strained $\{111\} /\{\overline{1} \overline{1} \overline{1}\}$ pyramidal islands also shows that the critical layer thickness somewhat depends on the island shape (see Fig. 5). However, we can still predict trends, e.g., for high growth temperatures (having a small island nucleation density) the critical layer is thinner. Our study also indicates that in case of a thinner critical layer, the island embryo should be larger than that for a thicker one. This can be understood in terms of a larger energy barrier which must be overcome by the embryo when nucleating on a thinner wetting layer.

Finally, we briefly comment on the ripening of the islands. When no more material is supplied by the wetting layer, the island density is no longer constant, because smaller islands will dissolve again. Allowing the island density to vary, we find that larger islands at a lower density are energetically preferred [see Fig. 4(a)]. Thus, our theory is in accord with the observed Ostwald ripening [10]. However, since noticeable changes in the island size and density resulting from ripening typically take many days, ripening is not important for device applications, where the islands are covered by a capping layer after a very short growth interruption, and was not observed in previous experiments performed on a shorter time scale.

In conclusion, we presented a novel explanation for the selection of particular sizes of self-assembled coherent islands in highly mismatched heteroepitaxy. It is possible to select the island size by changing the growth conditions and the nominal coverage. Our theory reproduces very well the experimental trends observed in the island growth. We have also shown how the critical layer thickness depends on growth conditions and settled this long-standing issue.

The authors thank E. Pehlke for fruitful discussions. This work was supported by Sfb 296 of the Deutsche Forschungsgemeinschaft.

*Present address: Department of Physics, Massachusetts Institute of Technology, Cambridge, MA 02139

[1] Y.W. Mo et al., Phys. Rev. Lett. 65, 1020 (1990).

[2] D. Leonard et al., Phys. Rev. B 50, 11687 (1994).

[3] J. M. Moison et al., Appl. Phys. Lett. 64, 196 (1994).

[4] R. Heitz et al., Phys. Rev. Lett. 78, 4071 (1997).

[5] N. Carlsson et al., Appl. Phys. Lett. 65, 3093 (1994).

[6] I. N. Stranski and L. von Krastanow, Akad. Wiss. Lit. Mainz Math.-Natur. Kl. IIb 146, 797 (1939).

[7] V. A. Shchukin et al., Phys. Rev. Lett. 75, 2968 (1995).

[8] I. Daruka and A.-L. Barabási, Phys. Rev. Lett. 79, 3708 (1997).

[9] C. Priester and M. Lannoo, Phys. Rev. Lett. 75, 93 (1995), and references therein.

[10] S. Lee et al., Phys. Rev. Lett. 81, 3479 (1998); S. H. Xin et al., Appl. Phys. Lett. 69, 3884 (1996).

[11] M. Bockstedte et al., Comput. Phys. Commun. 107, 187 (1997); http://www.fhi-berlin.mpg.de/th/fhimd/code.html

[12] Y. Chen and J. Washburn, Phys. Rev. Lett. 77, 4046 (1996).

[13] J. Tersoff and R. M. Tromp, Phys. Rev. Lett. 70, 2782 (1993).

[14] N. Moll et al., Phys. Rev. B 58, 4566 (1998).

[15] H. Yamaguchi and Y. Horikoshi, Phys. Rev. B 51, 9836 (1995).

[16] E. Pehlke et al., Appl. Phys. A 65, 525 (1997).

[17] D. Leonard et al., Appl. Phys. Lett. 63, 3203 (1993).

[18] G. S. Solomon et al., Appl. Phys. Lett. 66, 3203 (1995).

[19] J. G. Amar and F. Family, Phys. Rev. Lett. 74, 2066 (1995).

[20] H. T. Dobbs et al., Phys. Rev. Lett. 79, 897 (1997).

[21] A. Polimeni et al., Phys. Rev. B 53, R4213 (1996), and references therein. We take the experimental values for the coverage thicker than 1.5 ML.

[22] M. Berti et al., J. Vac. Sci. Technol. B 15, 1794 (1997).

[23] D. J. Bottomley, Appl. Phys. Lett. 72, 783 (1998).

[24] C. W. Snyder et al., Appl. Phys. Lett. 62, 46 (1993).

[25] Q. K. Xue et al., J. Vac. Sci. Technol. B 15, 1270 (1997).

[26] V. A. Shchukin et al., Surf. Sci. 352-354, 117 (1996). 